\title{
DEUX TYPES DE CELLULES “ROYALES» \\ CHEZ APIS MELLIFICA \\ EXISTE-T-IL CHEZ APIS MELLIFICA DES SEXUÉS DE REMPLACEMENT ?
}

PAR

\section{R. DARCHEN et M. VUILLAUME}

Station de Recherches apicoles, Bures-sur-Yvette

Iaboratoire de Biologie animale, SPCN, Faculté des Sciences, Rennes.

Après avoir introduit I 500 grammes d'abeilles sans reine dans une ruchette Dadant 4 cadres, nous les avons incitées à 1'élevage royal en leur présentant un cadre garni d'œufs pondus dans une autre ruche, au cours d'une nuit. Nous possédions ainsi, au départ, des œufs de même âge. Après apport de deux cadres de miel et d'un cadre nourrisseur quotidiennement empli de sirop de sucre, l'élevage royal a effectivement commencé. Mais nous avons détruit chaque jour les cellules royales à peine ébauchées. A notre grand étonnement, après les dernières destructions, le $7^{\mathrm{e}}$ jour après la ponte et les jours suivants, nous avons constaté la reprise de l'élevage sur des larves d'ouvrières très grosses, proches de l'operculation. Ces larves choisies par les abeilles, ayant reçu un important supplément de gelée, ont donné le $\mathrm{i} 6^{\mathrm{e}}$ jour, des reines apparemment normales : abdomen de $9 \mathrm{~mm}$, proboscis de $3,5 \mathrm{~mm}$, aiguillon légèrement recourbé, tête plus petite, mandibules dentelées ( 2 dents), pas de brosses sur les pattes postérieures ; fait important, les ovaires étaient plus gros que ceux des ouvrières pondeuses, mais légèrement moins volumineux que ceux des reines. Nous avons obtenu des fécondations de telles reines. Leur ponte donne un couvain d'ouvrières normal. Nous avons donc bien affaire à de véritables reines.

Or, on admet depuis I925 (travaux de ZANDER et BECkER) qu'il est impossible d'obtenir des reines après le $3^{\mathrm{e}}$ jour d'existence larvaire si l'élevage proprement royal n'a pas commencé avant.

Frappés par cette contradiction apparente, nous avons repris et poussé plus loin nos observations. Nous avons d'abord remarqué qu'au 
moment de la dernière destruction des cellules royales, certaines larves de quatre jours étaient logées dans des cellules d'ouvrières plus larges que les cellules normales et reposaient sur une quantité de gelée royale beaucoup plus importante que celle contenue dans les cellules voisines. Ces grandes cellules isolées avaient la forme d'un tronc de pyramide. Nous avons d'ailleurs retrouvé des cellules de ce type dans des ruches normales, non orphelines, au milieu du nid à couvain.

\section{Les hétérogénéités du régime larvaire.}

D'après l'étude anatomique très soignée exécutée par BECKER sur des larves dont on entreprenait l'élevage royal en les greffant jour par jour dans des ruches orphelines, il se produit entre le $3^{\mathrm{e}}$ et le $4^{\mathrm{e}}$ jour, en l'espace de I2 heures, une série de "transformations catastrophiques", dit-il, qui font régresser d'une manière irréversible les ovaires et les annexes génitales, alors que les glandes pharyngiennes, jusque-là très petites, se développent énormément. A partir du terme de trois jours et demi, l'élevage royal artificiel ne change rien à cette régression ni à ce développement; d'ailleurs les abeilles acceptent très difficilement les vieilles larves d'ouvrières introduites dans les cellules royales artificiellement, dit BECKER. Nous l'avons vérifié également; nous échouons même avec des larves royales prélevées dans les cellules royales $1 \mathrm{e} 7^{\mathrm{e}}$ jour après la ponte.

GoNTARSKI (I94I) signale qu'on tencontre fréquemment dans les ruches toutes les formes possibles de passage morphologique entre reines et ouvrières, mais il semble indiquer que ces formes ne surviennent qu'à la suite d'un élevage royal provoqué ou naturel. Dans un autre travail, GONTARSKI (I953) remarque la présence, à la suite d'un orphelinage, de cellules plus richement pourvues en gelée royale que d'autres. Mais d'après la photographie qui les représente ni leur forme, ni leur taille ne semblent aucunement affectées et elles se trouvent côte à côte, alors que les nôtres sont le plus souvent isolées. L'auteur n'a pas recherché si ces larves, nourries avec davantage de gelée royale, donnent ou peuvent donner des reines même après plus de trois jours de vie larvaire.

Des séries d'individus de taille variable sont également signalées par Gösswald chez les Fourmis (I954).

\section{Les pré-reines.}

Comment donc, une fois encore, expliquer la contradiction apparente entre nos résultats et l'impossibilité jusqu'ici reconnue de transformer en reines des larves d'ouvrières de plus de trois jours? Il nous semble 
qu'on le peut en admettant que les larves élevées dans des cellules spéciales à forme pyramidale n'ont pas subi le changement caractéristique de régime signalé par NeLSON, STCRTEVANT et I, INEBURG en I924 et qui se produit justement après le $3^{\mathrm{e}}$ jour. A ce moment, la gelée royale qui a servi jusqu'à ce stade d'aliment aux jeunes larves est remplacée par le "pain d'abeilles ", mélange mal défini de miel et de pollen. Ce brusque changement se place tout justement au monnent où BFCKER situe sa transformation brusque de potentialités de la larve qui ne peut plus devenir une reine.

Il faut remarquer que le changement des possibilités d'évolution 11'est peut-être pas toujours aussi brusque que le pensent ZANDER et BFCKer, si l'on en croit d'anciennes expériences de KLEIN (I904) qui élève des reines à partir de larves de I à 6 jours en obtenant tous les intermédiaires entre reines et ouvrières (mais nous n'avons pu consulter son travail dans l'original).

Les laries des grandes cellules recevraient de la gelée royale beaucoup plus longtemps, et leurs possibilités de virage ier's le stade royal resteraient ainsi inaltérées plus longtemps. Ce seraient en quelque sorte des pré-reines qui peuvent ne pas être utilisées : elles donnent sans doute alors les formes intermédiaires signalées par Gox'TARSK et que tous les apiculteurs connaissent bien, car elles constituent une grande gêne dans la recherche de la reine. Ces pré-reines "abandonnées", qui donneront des ouvrières, éclosent du I $S^{\mathrm{e}}$ au $2 \mathrm{I}^{\mathrm{e}}$ jour. Par la suite, les ouvrières pondeuses ne pourraient-elles pas provenir en grande partie de ces "intercastes "?

\section{Comparaison avec les Termites.}

Ne serions-nous pas en présence du phénomène signalé par Grassé et Norrot chez les Termites? A la suite de traumatismes provoqués à l'intérieur d'une colonie, il apparaît fréquemment des néoténiques. Ne provoquons-nous pas, nous aussi des traumatismes à l'intérieur de nos colonies quand nous leur demandons de maintenir en permanence des élevages royaux? Toutefois, même dans les ruches où nous n'avons pas entretenu en permanence ces élevages royaux, il existe de telles cellules de pré-reines. Chez les termites : l'existence d'intercastes nymphes-soldats semble indiquer que la lignée conduisant aux imagos ailés n'a pas perdu toute faculté d'évoluer en soldats " (NorRoT). Il signale, ainsi que d'autres auteurs, la présence d'individus aberrants, qui non seulement possèdent des ébauches alaires, mais montrent aussi une tête plus ou moins intermédiaire entre celle du soldat normal et celle d'une nymphe. Prckens, lui aussi, observe chez les 'Termites qu'entre les sexués ailés et les formes aptères, il existe, surtout dans les vieilles colonies, tous les intermédiaires. Entre les soldats et les ouvriers se trouvent quelquefois, 
dans des colonies nonvellement fondées, des "soldats-ouvriers " qui disparaitront par la suite. Des faits semblables sont également relevés par Buchur chez les Termites.

\section{Conclusions.}

Pour conclure, disons que les abeilles orphelines, dont on a détruit à plusieurs reprises les cellules royales, peuvent encore produire des reines

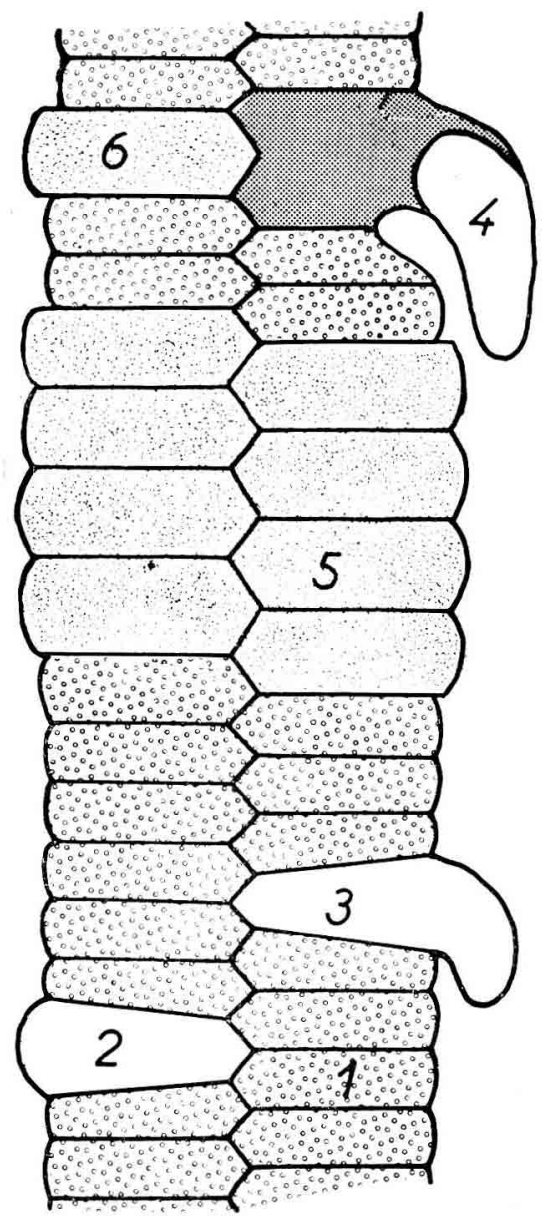

Fig. I. - No : Cellules d'ouvrieres normales. - Vo 2 : Cellule d'une ouvriere née d'une larve de pré-reine. - No 3 : c'ellule d'une reine née d'une larve de pré-reine on d'unc reine élévée à la

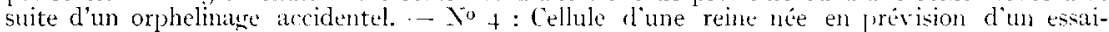

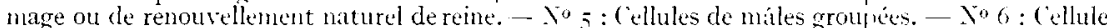
de mâle isolée.

à caractéristiques morphologiques normales bien après l'âge de trois jours de vie larvaire; ces reines fécondables proviennent de larves élevées normalement sur des cellules pyramidales élargies, qui reçoivent beaucoup 


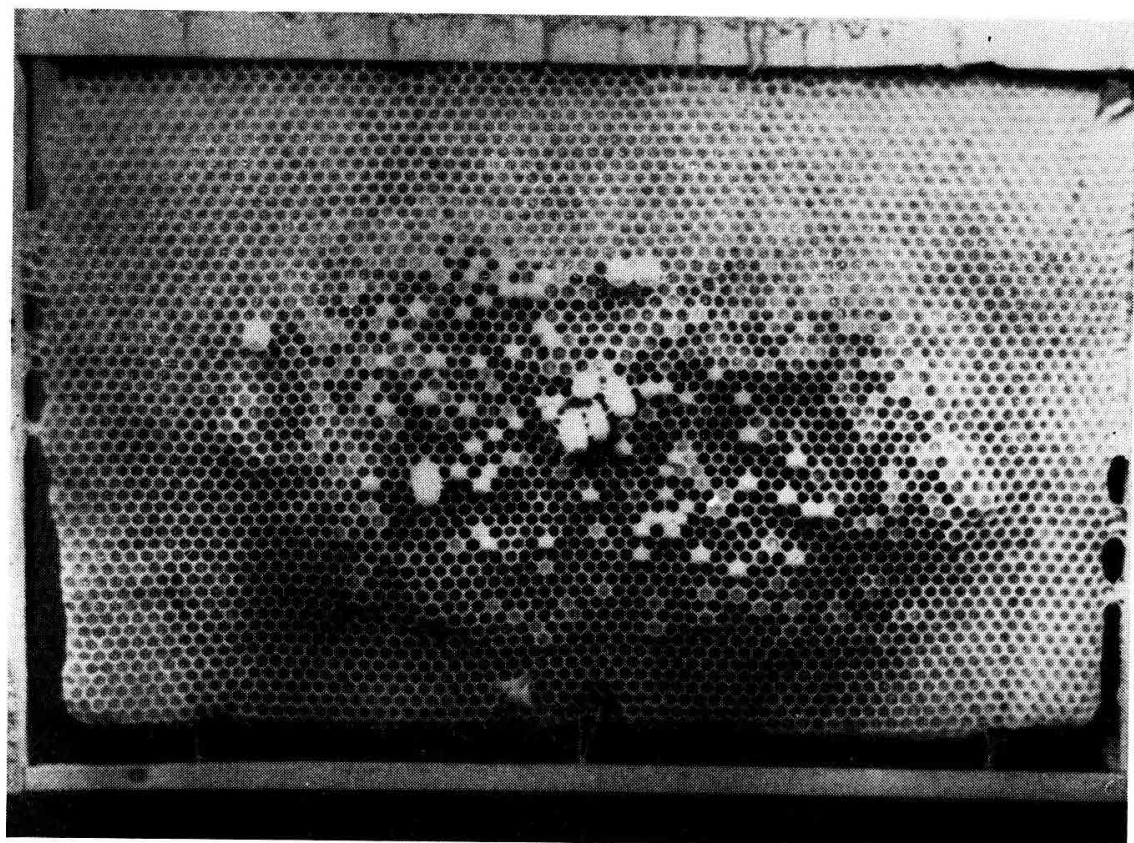

Piı́to i : Cadre de couvain avec cellules de reines el cellules operculées de pré-reines.

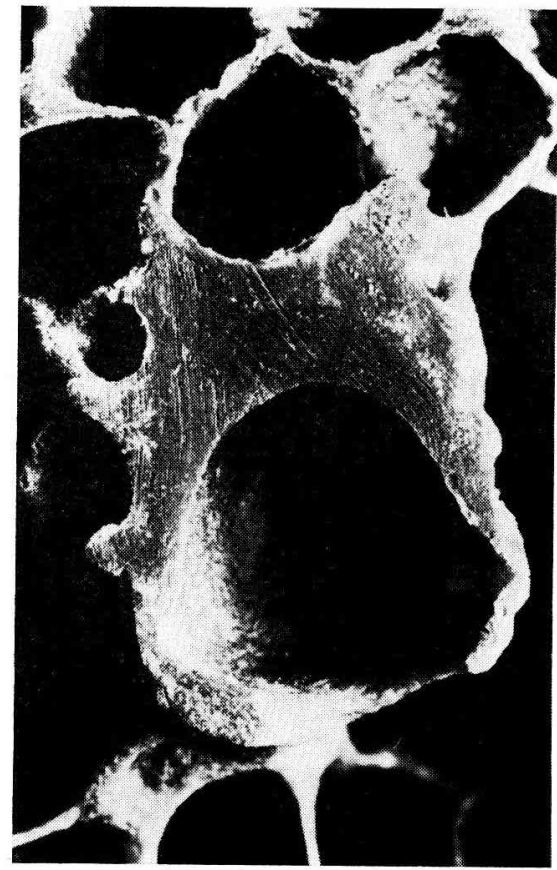

Photo 2 : Base d'une cellule royale en coupe, montrant la base de cire épaisse (voir texte).

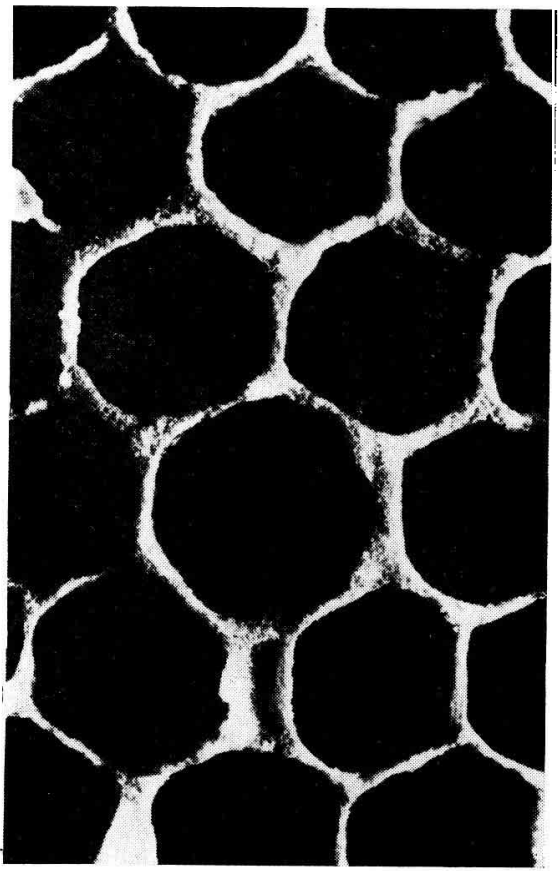

Proto 3 : Cellule de "pré-reine "au centre du rayon, parmi des cellules d'ouvrières. 
plus de gelée royale que les autres. Elles gardent ainsi, sans doute, plus longtemps leurs potentialités évolutives (fig. I).

Il y aurait en quelque sorte 2 modes d'élevage de reines chez les abeilles : dans un premier cas, une colonie élève spontanément une reine soit en vue d'un essaimage, soit en vue du remplacement d'une reine déficiente. Dans ce cas, les abeilles préparent des cellules rovales d'un premier type qui font hernie hors des rayons, et dont la base est parfaitement arrondie. Il est très fréquent de trouver des cufs dans ces ébauches de cellules royales, pendant la période d'essaimage, dans les fortes colonies. A l'éclosion de la larve, les abeilles étirent à partir de cette ébauche naturelle de cellule royale, une cellule royale typique, cylindrique (fig. I).

Dans un deuxième cas, celui d'une ruche orphelinée accidentellement et brusquement, les abeilles construisent des cellules royales à partir de cellules d'ouvrières. Ėlles élargissent alors la cellule d'ouvrière en tronc de pyramide et constituent en dehors du cadre "la hernie " cylindrique qui termine toutes les cellules royales, mais la base de la cellule reste polyédrique. Pendant cet élevage, les abeilles prépareraient des cellules de pré-reines (photo $11^{\circ} \mathrm{I}$, et fig. I).

Signalons enfin que les deux types de cellules royales sont nettement différentes. Tans le premier cas, elles reposent sur un bloc de cire massif (voir fig. I et photo $1^{\circ} 2$ ), sont cylindriques, sortent presque entièrement du rayon. Elles sont disposées presque toujours sur les bords du cadre ou au moins entre le nid à couvain et le nectar. Dans le second cas, elles prennent naissance au milieu du cadre et sont situées à l'intérieur du nid à couvain (photo no 3 ). Elles ont la forme d'un tronc de pyramide enfoui dans le rayon, recouverte sur la grande base par un chapeau cylindrique (no 3 sur la fig. I). Quant aux ouvrières naissant à partir des pré-reines, elles sont logées dans des cellules à structure spéciale également et facilement reconnaissables ( $n^{\circ} 2$ sur la figure). Operculées et vues de dessus, les cellules ressemblent exactement à des cellules de mâles isolées ( $11^{\circ} 6$ sur la fig. I et photo $n^{0}$ I), avec un opercule plus large que celui des cellules d'ouvrières normales, et bombé, dépassant la surface du couvain d'abeilles ouvrières. En coupe, elles sont nettement différentes des cellules de mâles $\left(n^{\circ} 5\right.$ et 6 sur la fig.) et d'ouvrières $\left(n^{\circ} \mathrm{I}\right)$. Elles correspondent à la partie du tronc de pyramide qui sert de base à la cellule royale. A ce tronc de pyramide, le "chapeau " des cellules royales n'est pas ajouté.

\section{RÉSUMÉ}

Dans cette étude, nous montrons que, contrairement à l'opinion générale, des larves âgées de plus de trois jours pouvent ètre transformées en reines; il existe, en outre, en permanence dans la ruche, des larves de substitution ou pré-reines. 


\section{SUMMARY}

In this paper, we are trying to prove that more than three days old larvae can become queens; substitution larvae or pre-queens are not permanently in the hive.

\section{ZLSAMMENFASSUNG}

In dieser Arbeit haben wir versucht die Frage zu klären ob über drei Tage ältere Larven dazu fähig sind Königinen zu werden und übrigens ob keine besondere Larven im Bienenvolk bestehen die als Vor-Königinen betrachtet werden können.

\section{RËFÉRENCES BIBLIOGRAPHIQLES}

Bechi, (H.). - Die Neotenie bei Reticutitermes. Ins. Soc., vol. 3, $1^{\circ}$ I, I956. Gontarski (H.). - Uber Zwischenformen von Königin und Arbeiterin im Staat der Honigbiene, A pis mellifica ; Z. Wiss. Zool., I54; 345-66, I94I. GoxTarski (H.). — Zur Brutbiologie der Honigbiene; Z. Bienenforsch. B. 2, I953.

GösswaLd (K.), Bitr (K.). - Untersuchungen zur Kastendetermination in der Gattung Formica ; Ins. Soc., I, no 4, 305-3I8, I954.

Grasse (P. P.), Noirot (Ch.). - La société de Calotermes flavicolis, de sa fondation an premier essaimage. C. R. Acad. Sc. (sous presse), I95S.

KLEIN. - Futterbrei und weibliche Bienenlarven; Bienenpflege, Lurlwigsburg, $26,5,1904$.

Neisox (A. J.), Sturtevint (A. P.), Lineburg (B.). - Growth and feeding of honeybee larvae. L. S. Dept. Agric. Dept. Bull., I222, I924.

Norror (Ch.). - Recherches sur le polymorphisme des 'lermites (Termitidae). Thèse Paris, Masson, Ann. Sc. Nat., I955.

Picklins (A. L.). - Links and gaps in the common castes of termites. Insectes sociaux, $3, n^{\circ} 2,233-8$, I956.

Zander (E.), Becker (F.). - Die Ausbildung des Geschlechtes bei der Honigbiene II. Erlanger Jahrg. Bienenk. B. 3, I6I-246, I925. 